\title{
Fortificación de alimentos con hierro y zinc: pros y contras desde un punto de vista alimenticio y nutricional
}

\section{Food fortification with iron and zinc: pros and cons from a dietary and nutritional viewpoint}

José BOCCIO'

Josefina Bressan MONTEIRO²

\section{RE S U M E N}

La deficiencia nutricional de ciertos minerales como el hierro y zinc afectan a la población a escala mundial con graves efectos sobre la salud dependiendo fundamentalmente del grado de incidencia y de la magnitud de la deficiencia. La fortificación de alimentos ha resultado ser la estrategia más efectiva para corregir esta situación con inconvenientes que radican no solamente en la elección del alimento a utilizar como transporte para dichos nutrientes sino también en la correcta elección del compuesto a utilizar como fortificante ya que en general aquellos compuestos que poseen una adecuada biodisponibilidad presentan cambios en las características sensoriales de los alimentos fortificados poco aceptables para su consumo. Los compuestos inertes, que poseen propiedades optimas desde del punto de vista tecnológico poseen una baja absorción que los convierte en compuestos nutricionalmente poco útiles. En este trabajo se discute con mayor detalle estos aspectos con el fin de llegar a un entendimiento más profundo de la problemática antes mencionada.

Palabras Claves: hierro, zinc, fortificación, alimento.

\section{A B S T R A C T}

The nutritional deficiency of essential minerals, such as iron and zinc, affects the population worldwide with different effects on human health. Food fortification has been proven to be one of the most effective strategies

\footnotetext{
${ }^{1}$ Laboratorio de Radioisótopos y Laboratorio de Isótopos Estables Aplicados a Biología y Medicina, Facultad de Farmacia y Bioquímica, Universidad de Buenos Aires. Junín 956. 1113, Buenos Aires, Argentina. E-mail: jboccio@ffyb.uba.ar

2 Departamento de Nutrição e Saúde, Universidade Federal de Viçosa, Av. PH Rolfs, s/n, 36570-000, Viçosa, MG, Brasil. Coorespondência para/Correspondence to: J.B. MONTEIRO. E-mail: jbrm@ufv.br
} 
to overcome this situation, although limitations residing not only in the choice of food to provide these nutrients but also in the correct selection of the fortification compounds are very important. In general, iron and zinc compounds, that have high bioavailability, lead to alterations in the sensorial properties of the fortified food, making it less acceptable for consumption. On the other hand, non-reactive compounds with excellent technological properties have very low bioavailability, which renders them of no value from a nutritional point of view. In the present article, fortification aspects are discussed in order to achieve a better understanding of this problem.

Index tems: iron, zinc, fortification, food.

\section{INTRO D U C C IÓ N}

La deficiencia de hierro es la carencia nutricional más frecuente en el ámbito mundial afectando en promedio al $30 \%$ de la población en general. Su incidencia en los países desarrollados es de aproximadamente del 10\% cifra que aumenta al $40 \%$ en los países que están en vías de desarrollo. La deficiencia de zinc es tan grave como la deficiencia de hierro. La deficiencia de este micronutriente afecta a la población que vive no solo en los países en vías de desarrollo sino también a aquellos que viven en los países desarrollados, afectando en consecuencia a la población mundial a escala global. Estudios basados en encuestas nutricionales han demostrado que la ingesta promedio de zinc a nivel mundial oscila entre un $50 \%$ a un $80 \%$ de los requerimientos diarios para este micronutriente ${ }^{1,2}$.

Los principales grupos de riesgo que poseen mayor probabilidad de sufrir deficiencia de hierro y zinc corresponden a aquellos grupos poblacionales en los que existe un inadecuado consumo y/o asimilación de hierro o zinc asociado generalmente a un aumento de su demanda. A estos grupos corresponden principalmente los lactantes, niños, adolescentes, embarazadas y mujeres en edad reproductiva. Sin embargo, es importante remarcar que la deficiencia de zinc a diferencia de la de hierro también puede afectar a ancianos, hombres adultos y mujeres posmenopáusicas ${ }^{3,4}$.

Las consecuencias de la deficiencia de estos micronutrientes sobre la salud de la población implican, entre otras, durante el embarazo, un aumento en la incidencia de partos prematuros llegando a producirse en los casos más graves, mortalidad materna y/o fetal. Los niños que nacen sin una adecuada cantidad de hierro en sus depósitos 0 aquellos que no tienen una ingesta adecuada de hierro y/o zinc durante su crecimiento pueden poseer un menor desarrollo intelectual y psicomotor con retardo en su crecimiento que dependiendo de las circunstancias puede llegar a ser de características irreversibles. En la persona adulta estas deficiencias cursan con disminución del rendimiento psicomotor e intelectual, produciendo una significativa reducción en la capacidad de trabajo y en consecuencia un descenso en la productividad, acarreando de esta manera serias consecuencias a nivel sanitario, social y económico en aquellos países donde estas deficiencias poseen altos índices de incidencia, agravando aún más la situación de la región ${ }^{3,-7}$.

Si bien resulta difícil evaluar el costo monetario que representa la tragedia personal y social de pérdidas humanas causadas por la deficiencia de hierro y zinc, el Banco M undial pudo estimar que las pérdidas causadas por la malnutrición de micronutrientes, en donde la deficiencia de hierro y zinc poseen la mayor prevalencia, representan en incapacidades y muertes, un costo del 5\% del Producto Bruto Interno (PBI) en aquellos países que se encuentran significativamente afectados por estas deficiencias nutricionales. Sin embargo, la solución a este problema, mediante la implementación de estrategias adecuadas como la fortificación de alimentos, tiene un costo económico inferior al 
0.3\% del PBI, representando una relación costobeneficio cercana a 20. Esto significa que por cada dólar invertido el la prevención de estas deficiencias nutricionales se evitan gastar 20 dólares para solucionar sus consecuencias ${ }^{8}$.

Las vías de solución y/o prevención a este problema nutricional, que han demostrado poseer la mejor relación costo/efectividad, han sido la fortificación de alimentos o la suplementación farmacológica con el o los micronutrientes deficientes. Ambos procedimientos poseen diferentes ventajas y desventajas, que fundamentalmente dependen de las propiedades fisicoquímicas y biológicas del nutriente a utilizar, como así también de las características y costumbres de la población a la cual se desee aplicar dicho procedimiento ${ }^{8-14}$.

La fortificación de alimentos ha demostrado ser la estrategia más efectiva. Esta consiste en el agregado de el o de los micronutrientes deficitarios a un alimento utilizado como carrier o transporte, el cual debe ser cuidadosamente seleccionado en función de los hábitos alimentarios de la población, como así también del grupo poblacional considerado de riesgo. La ventaja fundamental que posee este procedimiento consiste en que la población que está afectada por la deficiencia de uno o varios micronutriente/ s en particular incorporará una cantidad adicional del mismo a través de la dieta que habitualmente está acostumbrada a ingerir, sin que se modifiquen sus costumbres alimentarías. No obstante, la utilización de la fortificación de alimentos como procedimiento debe ser considerada como un método profiláctico o preventivo para combatir la deficiencia de micronutrientes, en especial en el caso del hierro y zinc, ya que las dosis de fortificación son generalmente una fracción de los requerimientos diarios contenidos en la porción del alimento, razón por la cual, este procedimiento debe ser considerado como una estrategia de mediano a largo plazo ${ }^{8-14}$.

Los compuestos que se utilizan para la fortificación de los alimentos, en general, son aquellos que aportan un hierro de tipo no hémico, o compuestos inorgánicos de zinc, por lo que es importante el tipo de compuesto que se va a usar, como así también el alimento que va ha ser utilizado como vehículo de transporte, ya que el mismo puede interferir con la absorción de estos elementos, disminuyendo en consecuencia sus biodisponibilidades 9 .

La elección del compuesto a utilizar debe basarse en la biodisponibilidad del mismo, también son importantes los cambios que produzca en las características sensoriales del alimento, además de su incidencia sobre el costo final del alimento fortificado $0^{9,15}$.

\section{FORT IF ICACIÓ N CON HIERRO}

Para el caso de la fortificación con hierro; los compuestos de hierro de elevada solubilidad como el sulfato ferroso y el gluconato ferroso aportan un hierro de alta biodisponibilidad, pero éstos tienen la desventaja de permitir que el hierro libre iónico interactúe con los componentes del alimento, produciendo cambios en las propiedades sensoriales del mismo. Este metal actúa catalizando los procesos oxidativos, produciendo en consecuencia la oxidación de los ácidos grasos insaturados y el enranciamiento de los lípidos del alimento. Este proceso catalítico de oxidación afecta a otros nutrientes como ser las vitaminas y los aminoácidos, disminuyendo significativamente el valor nutricional del alimento ${ }^{9,15}$.

Existen compuestos con una moderada solubilidad en agua, como el fumarato ferroso y el succinato ferroso, que si bien tienen una elevada biodisponibilidad, tienen el inconveniente de poder ser utilizados únicamente en alimentos sólidos y deshidratados, puesto que de utilizarlos en alimentos acuosos neutros, los mismos precipitan y además la fracción de hierro liberado por los mismos interactúa con el alimento produciendo las alteraciones antes mencionadas ${ }^{9,15}$.

Los compuestos como el ortofosfato férrico, el pirofosfato férrico y hierro elemental son 
compuestos de baja solubilidad, por lo tanto si bien no producen un cambio significativo en las características sensoriales del alimento, ni en su valor nutricional, tienen la desventaja de poseer una pobre absorción y en consecuencia una biodisponibilidad muy baja. Eso los convierte en compuestos poco útiles desde el punto de vista nutricional9,15.

Los compuestos de hierro protegidos surgen como consecuencia de la necesidad de utilizar compuestos que aporten un hierro con alta biodisponibilidad, como la que poseen los compuestos solubles en agua y que además posean una baja reactividad con la matriz nutricional, con el fin de ser tecnológicamente aptos para ser utilizados en los procesos industriales de fortificación de alimentos ${ }^{9,15}$.

La hemoglobina, es un compuesto de hierro naturalmente protegido, esta posee una elevada biodisponibilidad aún en presencia de los inhibidores de la absorción de hierro presentes en la dieta. Sin embargo, su principal desventaja es la de ser un compuesto intensamente coloreado lo que limita significativamente su uso en la fortificación de alimentos. Otra desventaja resulta de la dificultad de obtener la hemoglobina en condiciones higiénicas adecuadas para ser utilizadas en la fortificación de alimentos ${ }^{9,15}$.

El EDTA-Fe (III) también posee una elevada biodisponibilidad aún en presencia de los inhibidores de la absorción de hierro y es relativamente estable a los procesos industriales de elaboración y almacenamiento de los alimentos fortificados. Sin embargo, este compuesto ha demostrado poseer serias desventajas con relación a los cambios que produce en el color de ciertos alimentos cuando este es adicionado a los mismos; como así también el potencial aumento que podría producir en la absorción de ciertos metales tóxicos como consecuencia de la quelación de los mismos en el tracto gastrointestina ${ }^{9,15}$.

Los compuestos quelados a aminoácidos tienen como ventaja respecto al EDTA-Fe (III) de estar formados por componentes naturales que habitualmente se encuentran en los alimentos.
Sin embargo, no hay estudios concluyentes con respecto a su biodisponibilidad, a los cambios en las características sensoriales de los alimentos fortificados con estos compuestos, como así también de estabilidad de los mismos a los procesos industriales de producción a los que son so metidos estos alimentos fortificados ${ }^{9,16}$.

El sulfato ferroso estabilizado, es el sulfato ferroso microencapsulado, en el cual, el sulfato ferroso se encuentra protegido de su entorno por una membrana de fosfolípidos, evitando de esta forma la interacción con el alimento. Este compuesto de hierro protegido fue exitosamente utilizado desde hace varios años para fortificar leche fluida y productos lácteos por la industria alimentaria en Argentina, Australia e Israel ya que el mismo, a diferencia de los otros compuestos de hierro, fue concebido desde un principio para ser utilizado en la fortificación de este tipo de alimentos ${ }^{17}$.

El sulfato ferroso estabilizado ha sido evaluado intensamente en cuanto a sus propiedades nutricionales y de estabilidad a los procesos industriales, demostrando que posee una elevada biodisponibilidad, baja toxicidad y el mismo comportamiento metabólico que el hierro aportado por el sulfato ferroso, además de ser estable a los procesos tecnológicos de producción industrial de este tipo de alimentos. Sin embargo, este compuesto tiene el inconveniente de ser mucho más costoso que el sulfato ferroso no encapsulado, lo que en muchos casos podría ser un factor limitante en su uso masivo como fortificante alimentario ${ }^{17}$.

\section{FORTIFICACIÓ N} CON ZINC

Con respecto a la utilización de compuestos de zinc en fortificación de alimentos, la Food and Drug Administration (FDA) ha considerado, independientemente de la biodisponibilidad de los mismos, a cinco compuestos de zinc como GRAS para ser utilizados en la fortificación de alimentos, ellos son: sulfato 
de zinc, cloruro de zinc, gluconato de zinc, óxido de zinc y estearato de zinc ${ }^{9}$.

El óxido de zinc es comúnmente usado en la fortificación de alimentos. En los países industrializados este compuesto es ampliamente utilizado para la fortificación de cereales. Este es un polvo blanco de bajo precio, que no causa problemas organolépticos cuando es agregado en cantidades pequeñas a alimentos sólidos. Sin embargo, este compuesto posee una baja biodisponibilidad, lo que lo convierte en un compuesto poco útil desde un punto de vista nutricional ${ }^{9}$

El sulfato de zinc también es utilizado en la fortificación de alimentos, es mucho mejor absorbido que el óxido de zinc, pero es más costoso que este, además de producir cambios en las características sensoriales del alimento. También, se ha observado que dependiendo de la dosis este compuesto puede provocar nauseas ${ }^{9}$.

El citrato de zinc también ha sido utilizado en la fortificación de alimentos, pero con poco éxito, este compuesto es bien absorbido. Sin embargo posee un sabor muy fuerte que es muy difícil de disimular, razón por la cual resulta ser un compuesto poco útil para ser utilizado en la fortificación industrial de alimentos ${ }^{9}$.

Lamentablemente a diferencia de lo que ocurre en el caso de los compuestos de hierro, no se han desarrollado extensamente los compuestos protegidos de zinc. El único compuesto de zinc protegido desarrollado e investigado en la actualidad es el gluconato de zinc estabilizado con glicina. Este compuesto ha sido utilizado ampliamente por la industria Argentina para la fortificación de productos lácteos y en Australia para fortificar diferentes jugos de frutas. Este compuesto tiene importantes características para ser considerado en la fortificación de alimentos, ya que además de poseer una adecuada biodisponibilidad, no produce cambios en las características organolépticas de los alimentos fortificados lo que lo convierte en un compuesto útil tanto desde un punto de vista nutricional como tecnológico-industrial ${ }^{9,18}$.

\section{A L I M E N T O S}

Los alimentos usados como transporte 0 carrier para estos minerales deben reunir ciertos requisitos, siendo el principal de ellos el de ser ampliamente consumido por los grupos de riesgo. Los alimentos más utilizados para este fin son principalmente los cereales y los productos lácteos y en menor proporción la sal, el azúcar y los condimentos. Sin embargo, los estudios realizados por Dutra de Oliveira y colaboradores en Brasil demostraron la factibilidad de utilizar eficientemente el agua potable como vehículo de fortificación $n^{9,15,19,20}$.

Los cereales, las harinas de éstos y los productos alimenticios derivados de ellos, son los más frecuentes vehículos utilizados en la fortificación con hierro, zinc y otros nutrientes, ya que son uno de los alimentos más ampliamente consumidos por la población. Generalmente la cantidad de hierro y/o zinc agregada a los productos refinados de los cereales es muy baja, ya que habitualmente se les agrega la cantidad necesaria para llegar al valor que originalmente poseía el grano entero antes de su refinamiento. En el caso de la fortificación, las cantidades de estos minerales adicionadas deberán ser sustancialmente mayores, en general, del orden de los $55 \mathrm{mg}$ a $65 \mathrm{mg}$ por $\mathrm{kg}^{21}$.

Una de las principales desventajas de la utilización de las harinas como vehículo de transporte para el hierro y zinc radica en su alto contenido de ácido fítico, de hasta un $1 \%$ en el grano entero y de unos $100 \mathrm{mg}$ por $\mathrm{kg}$ en las harinas refinadas. Este posee un potente efecto inhibitorio sobre la absorción de estos minerales, disminuyendo consecuentemente su biodisponibilidad ${ }^{5,22}$.

Otra desventaja que posee la fortificación de harinas y cereales en el caso del hierro es su elevada susceptibilidad al enranciamiento, mediada a través de la oxidación catalítica de las grasas por este elemento. En general, con el fin de evitar este proceso se agregan compuestos inertes de hierro, como pirofostato férrico y hierro 
elemental, con la desventaja de que los mismos poseen una muy baja biodisponibilidad. Por esta razón es necesario el agregado de cantidades significativamente superiores de hierro, que en algunos casos son del orden de los $200 \mathrm{mg}$ a $500 \mathrm{mg}$ por $\mathrm{kg}$, juntamente con el agregado de un promotor de la absorción como el ácido ascórbico, ya que en caso contrario la utilidad de la fortificación puede ser considerada de dudosa efectividad ${ }^{15,23,24}$

Los productos lácteos al igual que los cereales y derivados de ellos son considerados como unos de los principales vehículos para ser fortificados con hierro, zinc y otros micronutrientes ${ }^{9,15}$.

La leche fluida de vaca, en muchos países es ampliamente consumida por los niños, uno de los principales grupos de riesgo. Las fórmulas infantiles son básicamente leche modificada de vaca y adicionadas con distintos nutrientes. Las leches chocolatadas también resultan un atractivo vehículo de fortificación ya que son muy apetecidas por los niños y adolescentes. La leche en polvo es distribuida en forma gratuita por muchos programas materno infantiles en algunos países en vías de desarrollo, por lo cual su fortificación con hierro y zinc debería ser imprescindible. Los yogures y productos fermentados, sobre todo aquellos dietéticos, son ampliamente consumidos por mujeres en edad reproductiva. Todo esto permite considerar que la fortificación con hierro y zinc de estos alimentos sería una herramienta esencial en la lucha contra la deficiencia de estos micronutrientes $5,9,25-27$.

Sin embargo, la fortificación de productos lácteos y sus derivados con un elemento como el hierro entraña diferentes dificultades propias de la compleja composición de este alimento y de la reactividad del hierro, ya que este metal interactúa con el mismo provocando la oxidación catalítica de las grasas, vitaminas y aminoácidos produciendo en consecuencia rancidez y disminución del valor biológico del alimento. En el caso del zinc, la utilización de algunos compuestos como el sulfato de zinc pueden provocar cambios en las características sensoriales del alimento fortificado, mientras que la utilización de compuestos inertes como el oxido de zinc puede precipitar depositándose en el fondo del recipiente siendo los mismos no ingeridos adecuadamente; además de poseer una biodisponibilidad muy limitada ${ }^{5,9,28}$.

Por otra parte, distintos nutrientes presentes en los productos lácteos, como el calcio, la caseína, las proteínas del suero y el cacao en el caso particular de las leches chocolatadas, podrían provocar una disminución significativa de la absorción del hierro y/o el zinc. Para el caso del hierro, el agregado de ácido ascórbico ha demostrado ser efectivo en solucionar en parte este efecto inhibitorio. Sin embargo la vitamina $C$ en medios líquidos, como la leche fluida, se degrada rápidamente a ácido dicetoglucónico, provocando importantes cambios en las características sensoriales del alimento 5,17,29-32.

También es necesario tener en cuenta que en muchos países la leche es consumida juntamente con otras infusiones como té, café, chocolate o mate que, en contacto con el hierro usado como fortificante de la leche, se torna de un color negro-grisaceo, condición que hace al alimento inaceptable desde el punto de vista de sus cualidades sensoriales ${ }^{15}$.

Lo anteriormente mencionado, por un lado demuestra la utilidad de este alimento como vehículo de fortificación con nutrientes como el hierro y el zinc, pero por otro lado, también evidencia el problema de su fortificación con elementos como estos como consecuencia de su elevada reactividad y/o cambios significativos en las características sensoriales. Es por ello, que la selección de los compuestos adecuados de estos metales resulta imprescindible para la fortificación de este alimento en particular y de todos los alimentos fortificados en general.

\section{CONCLUSIÓ N}

De esta forma podemos concluir que la fortificación de los alimentos es un eficiente 
procedimiento para prevenir la deficiencia nutricional de hierro y zinc. El uso de algunos compuestos de hierro y zinc nos permite fortificar en forma adecuada diferentes alimentos sin alterar sus propiedades sensoriales. Sin embargo estos compuestos deben ser cuidadosamente seleccionados de acuerdo a su biodisponibilidad, la composición de la matriz nutricional del alimento a fortificar, como así también de los procesos tecnológicos a utilizar durante los procesos industriales de fortificación y producción del alimento.

\section{REFERE N C I A S}

1. World Health Organization. Commission on Health and Enviroment. Report of the panel on food and agriculture. Geneva; 1992.

2. Calvo $E, G$ nazzo N. Prevalence of iron deficiency in children aged 9-24 mo from a large urban area of Argentina. Am J Clin Nutr 1990; 52:534-40.

3. Dallman P. Iron. Present knowledge in nutrition. 6th. ed. Washington (DC): ILSI; 1990. p.277-88.

4. Cousins R, Hempe J. Zinc: present knowledge in nutrition. 6th ed. Washington (DC): ILSI; 1990. p.289-308.

5. Salgueiro J, Zubillaga M, Lysionek A, Sarabia I, Caro $R$, De Paoli T et al. Zinc as an essential micronutrient: a review. Nutr Res 2000; 20:737-55.

6. Salgueiro J, Zubillaga M, Lysionek A, Cremaschi G, Sarabia I, Caro R, et al. Zinc status and immune system relationship: a review. Biol Trace Elem Res 2000; 76:193-205.

7. Salgueiro $M$, Zubillaga $M$, Weill $R$, Postaire $E$, Lysionek $A$, Caro $R$, et al. Is there a need of concern about zinc supplementation in diabetes mellitus patients? Biol Trace Elem Res 2001; 81:215-28.

8. Enriching L. Overcoming vitamin and mineral malnutrition in developing countries. Washington DC: The World Bank; 1994.

9. Salgueiro $M$, Zubillaga M , Lysionek A, Caro R, Weill $R$, Boccio J. Strategies to combat zinc and iron deficiency. Nutr Rev 2002; 60:52-8.
10. Allen L. Zinc and micronutrient supplements for children. Am J Clin Nutr 1998; 68:495-8.

11. Lofti M, Mannar V, Merx R, Naber-Van Den Heuvel P. Micronutrient fortification of foods. Current practices, research and opportunities. The Micronutrient Initiative. Ottawa: International Development Research Centre; 1996.

12. Schultink W, Ree M, Matulessi P, Gross R. Low compliance with an iron-supplementation program: a study among pregnant women in Jakarta, Indonesia. Am J Clin Nutr 1993; 57: 135-9.

13. Gibson R. Technological approaches to combating iron deficiency. Eur J Clin Nutr 1997; 51:25S-7S.

14. Gibson RS, Yeudall F, Drost N. Dietary interventions to prevent zinc deficiency. Am J Clin Nutr 1998; 68:484-7.

15. Hurrell R. Preventing iron deficiency through food fortification. Nutr Rev 1997; 55:210-22.

16. Fox T, Eagles J, Fairweather-Tait S. Bioavailability of iron glycine as a fortificant in infant foods. Am J Clin Nutr 1998; 67:664-8.

17. Boccio J, Zubillaga M, Caro R, Gotelli C, Gotelli M, Weill R. New procedure to fortify fluid milk and dairy products with high-bioavailable ferrous sulfate. Nutr Rev 1997; 55:240-6.

18. Salgueiro J, Zubillaga M, Lysionek A, Sarabia I, Caro $\mathrm{R}$, De Paoli $\mathrm{T}$, et al. Bioavailability, biodistribution and toxicity of Bio- $\mathrm{Zn}^{\mathrm{TM}}$ : a new zinc source. Comparative studies in rats. Nutrition 2000; 16:762-6.

19. Dutra De Oliveira J, Ferreira J, Vasconcellos V, $M$ archini J. Drinking water as an iron carrier to control anemia in preschool children in a day-care center. J Am Coll Nutr 1994; 13:198-202.

20. Dutra De Oliveira J, De Almeida C. Domestic drinking water-an effective way to prevent anemia among low socio-economic families in Brazil. Food Nutr Bull 2002; 23:213-26.

21. Darnton-Hill I, Mora J, Weinstein H, Wilburg S, Nalubola R. Iron and folate fortification in the Americas to prevent and control micronutrient malnutrition: an analysis. Nutr Rev 1999; 57: 25-31. 
22. Hallberg L, Brune M, Rosander L. Low bioavailability of carbonyl iron in man: studies on iron fortification of wheat flour. Am J Clin Nutr 1986; 43:59-67.

23. Hallberg L, Rossander L, Skanber GA. Phytates and the inhibitory effect of bran on iron absorption in man. Am J Clin Nutr 1987; 45:988-96.

24. Fomon S. Reflections on infant feeding in the 1970s and 1980s. Am J Clin Nutr 1987; 46: 171-82.

25.Davis A, Bolin T. Iron fortification of milk powder. Med J Australia 1976; 1:359-90.

26. Stekel A, Olivares M, Cayazzo M, Chadud P, Llaguno $S$, Pizarro $F$. Prevention of iron deficiency by milk fortification II. A field trial with a full-fat acidified milk. Am J Clin Nutr 1988; 47:265-9.

27. American Academy of Pediatrics. Iron fortification of infant formulas. Committee on Nutrition. Pediatrics 1999; 104:119-23.
28. Galdi M, Carbone N, Valencia M. Comparison of ferric glicinate to ferrous sulfate in model infant formulas: kinetics of TBA, lysine and methionine changes. J Food Sci 1989; 54:1230-3.

29. Hallberg L. Does calcium interfere with iron absorption? Am J Clin Nutr 1998; 68:3-4.

30. Emery T. Iron oxidation by casein. Biochem Biophysi Res Commun 1992; 182:1047-52.

31. Jackson L, Lee $K$. The effect of dairy products on iron bioavailability. Crit Rev Food Sc Nutr 1992; 31:259-70.

32. M inotti P, Buchonski S, Miller D. Effects of calcium supplementation, calcium source and lactose on iron absorption in the rat. Nutr Res 1993; 13: 1173-81.

Recebido para publicación en 4 de junio de 2002 y aprobación en 9 de abril de 2003. 